\title{
Educación formal y no formal. Un punto de encuentro en educación musical
}

\author{
Noemy Berbel Gómez ${ }^{\mathrm{a}, *}$ y Maravillas Díaz Gómez \\ aniversitat de les Illes Balears, España \\ ${ }^{\mathrm{b}}$ Universidad del País Vasco, España
}

INFORMACIÓN DEL ARTÍCULO

Historia del artículo:

Recibido el 3 de julio de 2013

Aceptado el 11 de diciembre de 2013

Palabras clave:

Educación formal-no formal

Complementariedad

Educación primaria

Escuelas de música

\section{RESU M EN}

Ámbitos educativos diferenciados, educación formal y no formal, pueden y deben interrelacionarse en beneficio de una formación integral del individuo al disponer de muchos más espacios para educar que las propias aulas (Mok, 2011). El texto pone de manifiesto la necesidad de coordinación entre el profesorado de música de los centros de enseñanza en la escuela primaria (educación formal) y el profesorado de escuelas de música (educación no formal), a partir de dos investigaciones enmarcadas en diferentes ámbitos territoriales y distanciadas por una década en el tiempo. Se vinculan los resultados obtenidos en los dos estudios al evaluar si el interés del profesorado por realizar un trabajo conjunto, recogido en la investigación 1, se manifiesta en el ámbito territorial de la investigación 2 y se ha implementado en su ámbito de estudio. Ambas investigaciones muestran la complementariedad existente entre estos dos ámbitos educativos y destacan la importancia de coordinarse, en aras de un óptimo aprovechamiento de los recursos disponibles, con el propósito de avanzar hacia una educación musical de calidad. El presente estudio concluye en que a pesar del avance experimentado en una década (2001-2012), todavía no se ha trasladado de una forma evidente a la realidad educativa.

(c) 2013 Instituto de Ciencias de la Educación de la Universidad de Oviedo. Publicado por Elsevier España, S.L. Todos los derechos reservados.

\section{Formal and non-formal education. A meeting point in music education}

\author{
A B S T R A C T
}

Different educational settings, formal and non-formal education, can and should interact for the benefit of a comprehensive training of the individual, so that there are many more spaces to educate them in than just classrooms (Mok, 2011). This article highlights the need for coordination among music teachers in primary schools (formal education) and teachers in music schools (non-formal education), using two research studies carried out in different territorial areas and separated by a decade in time. The results of these two studies are connected in that they evaluate whether teachers are interested in working together, described in investigation 1, and appears in the scope of investigation 2, and whether it has been implemented in its field of study. Both studies show the complementarity of these two educational settings, and emphasize the importance of coordination, for the sake of an excellent use of the available resources, in order to move towards quality in music education. This study concludes with the idea that, despite the advances experienced in the last decade (2001-2012), it has not obviously been transferred into an educational reality.

(c) 2013 Instituto de Ciencias de la Educación de la Universidad de Oviedo. Published by Elsevier España, S.L. All rights reserved.

\section{Introducción}

El reconocimiento por parte de las instituciones educativas hacia la educación musical quedó garantizado en España con la promulgación de la Ley Orgánica 1/1990, de 3 de octubre, de Ordenación

\footnotetext{
*Autor para correspondencia: Noemy Berbel Gómez. Universitat de les Illes Balears. Cra. de Valldemossa, km 7,5. CP 07122 Palma de Mallorca (España). Correo electrónico: noemy.berbel@uib.es
}

General del Sistema Educativo (LOGSE). Esta ley supuso un cambio cualitativo sustancial en la ordenación educativa de las enseñanzas musicales en el Estado español al incorporar al currículum la asignatura de música en los niveles de infantil, primaria y secundaria. En cuanto a las enseñanzas especializadas de música, marcó una distinción entre la enseñanza que se imparte en los conservatorios y la que se lleva a cabo en las escuelas de música. Por ello, en el contexto de nuestro estudio cabe destacar de dicha ley dos aportaciones relevantes. 
En primer lugar, con el fin de asegurar la adecuada preparación del profesorado que imparte las enseñanzas musicales en la etapa primaria, se crea la figura de maestro especialista de música, inexistente hasta la implantación de dicha ley. Esta especialización corre a cargo de las universidades.

En segundo lugar, se apuesta por las escuelas de música como centros de enseñanza no formal. Estos centros educativos ofrecen una formación musical de calidad, donde los aficionados pueden cursar estudios musicales sin límites de edad. El referente de España es el modelo europeo de escuelas de música al estar integradas en la Unión Europea de las Escuelas de Música (EMU).

Estas significativas contribuciones que introdujo la reforma educativa al establecer dos ámbitos educativos diferenciados (educación formal y no formal) pueden y deben interrelacionarse en beneficio de una educación musical más cohesionada y fortalecida. Se parte de la convicción de que no existe un solo modelo de educación sino varios, y que todos ellos contribuyen a la formación del individuo. En la actualidad, el universo educativo reconoce tres modalidades que intervienen en los procesos de aprendizaje: formal, no formal e informal. Esto lleva a considerar la necesidad de coordinación entre educación formal y no formal, sin obviar que la enseñanza/aprendizaje de la música debe tener en cuenta los conocimientos y experiencias adquiridas en contextos informales (Ibarretxe y Díaz, 2008; Mok, 2011). Según Coombs y Ahmed (1974, p. 27) la educación formal hace referencia al "sistema educativo altamente institucionalizado, cronológicamente graduado y jerárquicamente estructurado, que se extiende desde los primeros años de la escuela primaria hasta los últimos años de la universidad", mientras que la educación no formal se basa en "toda actividad organizada, sistemática, educativa, realizada fuera del marco del sistema oficial, para facilitar determinadas clases de aprendizaje a subgrupos particulares de la población, tanto adultos como niños". Numerosos autores han centrado sus estudios en el campo de la educación no formal: Coleman y Husen (1989), Rogers (2004), Torres y Pareja (2007), Touriñán, Rodríguez y Olveira (2005), Trilla (1998). Ambos tipos de educación, formal y no formal, comparten intencionalidad pedagógica, la organización y la sistematización (Riaño, 2008). Frente a estas dos modalidades se encuentra la educación informal, entendida, según Trilla (1993, p.17) como el "conjunto de procesos y factores que generan efectos educativos sin haber estado expresamente configurados a tal fin".

La relación entre las diferentes formas de aprender música ha estado entre las preocupaciones de los educadores musicales en las últimas décadas. Los estudios sobre educación formal e informal en el ámbito de la educación musical han sido ampliamente discutidos por diversos investigadores (Campbell, 1998; Hentschke, 2003; North y Hargreaves, 2008). Asimismo, la posible integración de caminos formales e informales en el aprendizaje de la música ha sido objeto de estudio por Cabedo y Díaz (2013), Colwell y Richardson (2002), Cremades y Herrera (2010), Oliveira (2003), Veblen y Olsson (2002), Waldron (2009), White y Geisler (2007). Sin embargo, a la hora de profundizar en la relación entre la educación formal y no formal nos encontramos con escasos estudios y con el requerimiento de entender cómo pueden converger ambos campos de influencia.

Algunas de estas investigaciones que relacionan la educación musical formal y no formal se abordan desde diversos ámbitos. Desde el ámbito universitario, Riaño (2010) describe un trabajo de investigación que se centró en la actividad musical gestionada a través de los vicerrectorados correspondientes de un grupo de universidades españolas: el G-9. El conocimiento de estas actividades, a partir de un enfoque etnográfico, permitió indagar acerca de la importancia que cada universidad otorga a la música y conocer si existe colaboración entre las universidades pertenecientes al Grupo, los planes y las políticas de gestión llevadas a cabo en favor de una calidad educativa. Del mismo modo, permitió comprobar si existen contextos de educación formal y no formal en la vida musical y cultural universitaria.
En el ámbito de las enseñanzas especializadas de música, Morant (2013) analizó los motivos por los que las escuelas de música de las sociedades musicales valencianas se comportan como centros educativos formales, al reproducir los planteamientos de los conservatorios de referencia, y propone acciones que puedan mejorar el modelo de centros no formales. La investigación se planteó mediante una metodología integrada de análisis con el uso de técnicas cuantitativas y cualitativas.

Y desde un enfoque interdisciplinario de la educación artística, Botella, Fernández y Minguez (2012) presentaron un proyecto integrador de las diversas artes (literatura, música, artes visuales y expresión corporal) a partir de la ópera, como herramienta para llevar a cabo un trabajo por competencias. El enfoque competencial ayuda a unificar los criterios de una metodología abierta e interdisciplinaria en la enseñanza de las artes y permite integrar el aprendizaje formal e informal en ellas.

La necesidad de indagar en la complementariedad de la educación formal (escuela primaria) y la educación no formal (escuelas de música) en el área de música es la que lleva al presente estudio. A tal efecto, los objetivos del mismo se concretan en: obtener evidencia acerca de la importancia que concede el profesorado a la organización y realización de acciones conjuntas entre los centros de enseñanza primaria (educación formal) y escuelas de música (educación no formal) y vincular los resultados de dos investigaciones que indagan sobre la posible colaboración entre los centros de educación formal y no formal con propuestas de coordinación entre ambos centros educativos (formal y no formal).

\section{Método}

\section{Diseño}

Se parte de las investigaciones de Díaz (2001) investigación 1 y Berbel (2012) investigación 2 relacionadas con la educación formal que reciben los alumnos de la escuela primaria (6-12 años) en la asignatura de música y la educación no formal que se imparte en las escuelas de música a niños de esta franja etaria. Dos son los intereses que nos conducen al presente estudio: la visión integradora que a partir de los objetivos y contenidos se desprende de los decretos curriculares de ambos centros educativos y conocer algunas de las dificultades por las que atraviesa en el ejercicio de su docencia el profesorado de música. Se pretende establecer puentes de comunicación a través de experiencias comunes de intervención entre el profesorado y el alumnado.

La investigación 1 "La música en la educación primaria y en las escuelas de música: la necesaria coordinación. Análisis para futuros planes de intervención educativa en la Comunidad Autónoma Vasca (CAV)" (Díaz, 2001) analizó el interés del profesorado por realizar acciones conjuntas entre los centros de primaria y las escuelas de música. El propósito de esta investigación fue conocer el interés de los especialistas de música de dos tipos de centros educativos claramente diferenciados (educación formal y no formal) por cooperar en proyectos de grupo compartidos. El estudio reflejó el grado de implicación del profesorado consultado y evidenció el deseo de colaboración conjunta.

La investigación 2 "La educación musical elemental en las Illes Balears (IB): evaluación de la calidad de las escuelas de música”, realizada por Berbel (2012), se llevó a cabo en la Comunidad Autónoma de las Illes Baleares. Esta investigación indagó sobre la calidad de las escuelas de música, a partir del establecimiento de unos indicadores de calidad, con el fin de analizar su impacto social, cultural y educativo y poder dar herramientas que permitan reforzarlo. El estudio manifestó las fortalezas y debilidades de las escuelas de música con el fin de concretar diversas propuestas de mejora para hacer frente a sus puntos débiles. 
En el presente trabajo se vinculan los resultados obtenidos en ambas investigaciones. La investigación de Díaz (2001) evidencia el acuerdo del profesorado por coordinarse en trabajos conjuntos en los centros de educación formal (escuela primaria) y no formal (escuela de música). La investigación de Berbel (2012) se vincula con los resultados obtenidos por Díaz (2001) al evaluar, en el contexto de su estudio, si el interés del profesorado por realizar un trabajo conjunto que recoge la investigación 1 se manifiesta de igual modo en el ámbito territorial de la investigación 2 y si este interés por coordinarse se traslada de una forma efectiva a los centros educativos una década más tarde.

\section{Participantes}

\section{Investigación 1}

Los participantes en la investigación 1 son, en primer lugar, el profesorado especialista de educación musical de la Comunidad Autónoma Vasca. De la población total de 487 profesores se recogió una muestra de 160 docentes, los cuales representan el 32,85\% de la población. Y en segundo lugar, los directores y profesores de las escuelas de música de dicha comunidad autónoma. Del total de la población de centros, 75 centros, se obtuvo una muestra de 22 centros, el $29,34 \%$ de la población, ascendiendo el número de cuestionarios recogidos a 154 .

\section{Investigación 2}

La población de la investigación 2 está formada por la totalidad de escuelas de música y danza reconocidas por la Consejería de Educación y Cultura de la Comunidad Autónoma de las Illes Balears y los centros autorizados de música, en total 42 centros. La muestra obtenida, una vez facilitado el cuestionario dirigido a los directores de la totalidad de centros, fue de 41 escuelas de música, un 97,62\% del total de la población.

\section{Instrumentos de evaluación}

\section{Investigación 1}

El instrumento de recogida de información seleccionado fue un triple cuestionario. Se partió de un estudio preliminar basado en los principios de investigación-acción, llevándose a cabo una intervención educativa. Posteriormente se realizó una preencuesta abierta para recoger opiniones que sirvieran de guía para la formulación definitiva del cuestionario. El cuestionario, de propia creación, fue revisado por cuatro expertos con los siguientes perfiles: psicología de la educación, didáctica general, didáctica de la música y asesor de un centro de profesores. El cuestionario presentado a los directores de escuelas de música constaba de 52 preguntas; 37 preguntas para el profesorado de estas escuelas y 32 para el profesorado de música de la escuela primaria.

\section{Investigación 2}

Para llevar a cabo la evaluación se estableció un sistema de indicadores de calidad, formado por un total de 75 indicadores y estructurado en 12 bloques, a partir del cual se construyó un cuestionario que permitiera recoger la información necesaria para evaluar cada uno de los indicadores, formado por 79 preguntas. Este sistema de evaluación (indicadores de calidad-cuestionario) de creación propia fue validado por un comité de once expertos de diferentes ámbitos: cuatro de gestión, cinco de educación y dos de métodos de investigación.

Además del cuestionario se llevaron a cabo, a lo largo de todo el proceso de recogida de datos, diversas entrevistas personales, por teléfono y por correo electrónico, con los directores de las escuelas de música. Y una vez recogida toda la información también se realizaron entrevistas con los directores para concretar y completar la información aportada a través de los cuestionarios.

\section{Procedimiento}

\section{Investigación 1}

La investigación se realizó en dos fases. La primera fase se enmarcó bajo los presupuestos teóricos y metodológicos de la perspectiva cualitativa a través de la investigación-acción y se llevó a cabo con el profesorado de escuelas de música de la localidad de Ondarroa (Comunidad Autónoma Vasca) y el profesorado de música de una escuela primaria de dicha localidad (Díaz, 1998). El trabajo conjunto del profesorado de ambos centros permitió constatar: a) la estrecha relación entre objetivos y contenidos de educación musical en ambos centros, más allá del diferente grado de especificidad "musical" de cada uno; $b$ ) que las actitudes hacia la música se ven fuertemente influidas por las expectativas que despiertan los profesores a través de las distintas propuestas y los diferentes planteamientos en las actividades programadas; $c$ ) que la relación entre el profesorado de escuelas de música y maestros de música de la escuela primaria puede ser favorecida, lo que suscitaría expectativas positivas del alumnado hacia el aprendizaje, y $d$ ) las experiencias compartidas con otros niños que hacen música ayuda al desarrollo de actitudes favorables y al despertar de nuevos intereses hacia el hecho musical.

La segunda fase de la investigación, de carácter cuantitativo, brindó información del profesorado a través de un cuestionario en el que se tuvieron en cuenta los datos de la primera fase. Participaron directores y profesores de la escuela primaria y escuelas de música de la Comunidad Autónoma Vasca.

El cuestionario se dirigió a los colectivos indicados por correo junto con una carta explicativa acerca de las finalidades de la investigación que se iba a realizar. El período de recogida de datos se llevó a cabo durante el mes de marzo de 2001.

\section{Investigación 2}

En el planteamiento general de la investigación de Berbel (2012) se observan dos fases. La primera fase fue descriptiva, basada en un método de análisis de documentos e información primaria (normativas, documentos de organización de centros y de archivo, memorias, etc.), y una segunda fase evaluativa, sustentada en el método de encuesta, con una técnica de cuestionario web autocumplimentado, completado con asesoramiento personal por parte de la investigadora y entrevistas personales. En esta segunda fase se concretó un sistema de indicadores de calidad, como herramienta para evaluar o analizar la situación de las escuelas de música, no solamente desde una perspectiva cuantitativa, sino también cualitativa.

Para elaborar el sistema de indicadores se partió del análisis de diversos trabajos, investigaciones y modelos de evaluación de reconocida valía por la comunidad científica como son el modelo Deming (1989), modelo EFQM de excelencia desarrollado por la European Foundation for Quality Management (1999) y su posterior adaptación a los centros educativos llevada a cabo por el Ministerio de Educación y Cultura (2001), y el modelo CIPP de Stufflebeam y Shinkfield (1989). Posteriormente se adecuaron estos indicadores a las escuelas de música y se elaboró un cuestionario, dirigido a la totalidad de los directores de los centros, con la pretensión de evaluar estos indicadores.

La recogida de datos se llevó a cabo a través de una plataforma web, diseñada para el estudio. Antes del lanzamiento se realizaron varias pruebas piloto, con la colaboración de algunos directores de las escuelas de música. En la web, además de acceder al cuestionario, podían consultar varios documentos relacionados con la investigación, como son: la presentación del estudio, la carta de agradecimiento por participar, el listado de centros participantes, información previa e instrucciones para el acceso y registro del cuestionario. También se incluyó un buzón de sugerencias, a través del cual podían hacer las aclaraciones que consideraran oportunas a fin de completar la información que registraron a través del cuestionario. El período de recogida de datos, en el cual los directores de las 
escuelas de música cumplimentaron los cuestionarios, fue de junio de 2010 a enero de 2011.

Para facilitar y concretar la aplicación y análisis del instrumento de evaluación (indicadores - cuestionario) se construyó una batería de indicadores donde se especificaba: cada uno de los indicadores, su descripción, la fórmula de cálculo que se debía aplicar, la escala aplicada en cada uno de ellos, la pregunta o preguntas del cuestionario relacionadas con el indicador que aportaban la información necesaria para su análisis y las observaciones oportunas.

\section{Análisis de datos}

Para hacer el análisis de los datos se utilizó en ambos casos el programa estadístico Statistical Package for the Social Sciences (SPSS) para Windows, versión 10, en la investigación 1, y versión 19 en la investigación 2.

Posteriormente, en las dos investigaciones se exportaron los datos a Excel con el fin de elaborar gráficos representativos de los resultados obtenidos. En el caso de la investigación 2 permitió revisar los datos recogidos a través de la plataforma web. Se calcularon los estadísticos para comprobar si las diferencias obtenidas en el resultado eran significativas o no. Asimismo se llevó a cabo un análisis de conglomerados o análisis de cluster de las medias con el fin de identificar grupos homogéneos, en relación con las características más relevantes, y el criterio usado para medir similitudes en el interior de cada cluster fue el de distancias. El procedimiento utilizado para definir los clusters fue el método de modelos no jerárquicos, dados las características y el número de participantes analizados en ambos estudios.

\section{Resultados}

De los aspectos comunes analizados en las dos investigaciones, que establecen un vínculo de unión entre ambas, se desprenden los siguientes resultados.

\section{Investigación 1}

Del análisis e interpretación de los datos obtenidos del cuestionario dirigido a los directores y profesores de las escuelas de música y de las escuelas de primaria de la investigación 1 , presentados en la tabla 1, se destaca un amplio acuerdo entre el profesorado de las escuelas de música y los maestros que imparten música en la escuela primaria en llevar a cabo trabajos conjuntos, en la medida que los centros faciliten dicha colaboración (81,2\% del profesorado de las escuelas de música y $98,5 \%$ del profesorado de la escuela primaria). Y del mismo modo, hay acuerdo en que los centros deben arbitrar soluciones para realizar proyectos en común, ya que la predisposición de las escuelas de música y escuelas de primaria es escasa a la hora de realizar proyectos comunes (49,9\% del profesorado de las escuelas de música y 51,9\% del profesorado de la escuela primaria).

Asimismo, los maestros de música de la escuela primaria y los profesores de escuelas de música están en desacuerdo en que la existencia de puntos en común entre la enseñanza de la música en la escuela primaria y la que se imparte en las escuelas de música no es prioritaria porque las finalidades son distintas, así lo manifiesta el $50,0 \%$ del profesorado de las escuelas de música y el 60,0 \% del profesorado de la escuela primaria.

Por último, como puede apreciarse en la tabla 1 se evidencia un amplio interés de los maestros de música y profesorado de escuelas de música por relacionarse en proyectos comunes tales como: organizar conciertos, conciertos didácticos y talleres; intercambiar materiales y recursos didácticos como libros, vídeos, $C D$, instrumentos; organizar charlas, debates, seminarios; establecer un plan de mejora social y educativo; elaborar programas y/o materiales didácticos de forma conjunta; y crear coros y orquestas con responsabilidad compartida.

\section{Investigación 2}

En la tabla 2 se recogen los resultados de la investigación 2 que se vinculan con el objeto de estudio de la investigación 1. En primer lugar, se evidencia si el interés del profesorado por realizar un trabajo conjunto que recoge la investigación 1 se manifiesta en el ámbito territorial de la investigación 2 . El 78,0\% de las escuelas de música consideran necesaria una coordinación entre el profesorado de ambos tipos de centros.

En segundo lugar, si este interés del profesorado por coordinarse, recogido en ambas investigaciones, se traslada de forma efectiva a los centros educativos. Del análisis de los indicadores de la investigación 2 relacionados con la coordinación entre las escuelas de música y los centros de primaria se destaca el porcentaje de centros que lle-

Tabla 1

Porcentaje de profesores y directores de escuela de música y escuela primaria que manifiestan interés en realizar acciones conjuntas

\begin{tabular}{|c|c|c|}
\hline Investigación 1 & Escuelas de música $(\mathrm{n}=154)$ & Escuela primaria $(\mathrm{n}=160)$ \\
\hline Interés del profesorado por realizar trabajos conjuntos & $81,2 \%$ & $98,5 \%$ \\
\hline Acuerdo en que los centros deben arbitrar soluciones para realizar proyectos en común & $49,9 \%$ & $51,9 \%$ \\
\hline Desacuerdo en que no existen puntos en común en la enseñanza & $50,0 \%$ & $60,0 \%$ \\
\hline \multicolumn{3}{|l|}{ Interés por relacionarse en proyectos comunes: } \\
\hline Organizar conciertos, conciertos didácticos y talleres & $96,2 \%$ & $99,4 \%$ \\
\hline Intercambiar materiales y recursos didácticos como libros, vídeos, $C D$, instrumentos & $91,6 \%$ & $95,6 \%$ \\
\hline Organizar charlas, debates, seminarios & $87,0 \%$ & $91,2 \%$ \\
\hline Establecer un plan de mejora social y educativo & $67,6 \%$ & $86,2 \%$ \\
\hline Elaborar programas y/o materiales didácticos de forma conjunta & $57,8 \%$ & $82,5 \%$ \\
\hline Crear coros y orquestas con responsabilidad compartida & $57,2 \%$ & $68,8 \%$ \\
\hline
\end{tabular}

Tabla 2

Porcentaje de directores de escuela de música que manifiestan interés en llevar acciones conjuntas con la escuela primaria

\begin{tabular}{|c|c|c|}
\hline Investigación $2(\mathrm{n}=41)$ & Sí & No \\
\hline Directores que consideran necesaria la coordinación & $78,0 \%$ & $21,0 \%$ \\
\hline Escuelas de música en las que existe coordinación con la escuela primaria & $36,1 \%$ & $63,9 \%$ \\
\hline Actividades dirigidas a la escuela primaria & $41,3 \%$ & $58,7 \%$ \\
\hline Proyectos conjuntos con otras instituciones & $58,5 \%$ & $41,5 \%$ \\
\hline Escuelas de música en las que el equipo directivo participa en foros de trabajo & $68,0 \%$ & $32,0 \%$ \\
\hline Escuelas de música que evalúan las colaboraciones externas realizadas & $25,0 \%$ & $75,0 \%$ \\
\hline
\end{tabular}


van a cabo las siguientes acciones: el 36,1\% de las escuelas de música llevan a cabo una coordinación con las enseñanzas musicales impartidas en la escuela primaria, frente a un 63,9\% que no la llevan a cabo. En cuanto a las escuelas de música que realizan actividades que favorecen la colaboración entre el profesorado de ambos tipos de centros, el 41,3\% de las escuelas de música organizan actividades dirigidas a la escuela primaria y el $58,5 \%$ llevan a cabo proyectos conjuntos con otras instituciones.

El 68,0\% del equipo directivo de las escuelas de música afirma que participa en encuentros y actividades con otros centros educativos, aunque solamente el $25,0 \%$ de las escuelas de música evalúan la gestión e incidencia de las colaboraciones externas realizadas.

\section{Discusión y conclusiones}

El interés de los maestros de música por relacionarse con el profesorado de escuelas de música en la organización de proyectos conjuntos se hace evidente en los resultados anteriormente expuestos en la investigación 1 . Se destaca una mayor predisposición de colaboración por parte de los maestros de música de la escuela primaria que por parte del profesorado de escuelas de música, aunque se considera que el grado de implicación de éstos también es muy elevado.

De las seis posibilidades de intercambio ofrecidas en el cuestionario, se sitúa en primer lugar, el deseo de organizar conciertos, conciertos didácticos y talleres. Le sigue en orden de importancia la necesidad de intercambiar materiales y recursos didácticos como libros, vídeos, discos, instrumentos. En tercer lugar, organizar de forma conjunta, charlas, debates y seminarios. Establecer un plan de mejora social y educativo se sitúa en cuarto lugar. El quinto lugar corresponde a la elaboración de programas y/o materiales didácticos de forma conjunta. Finalmente, crear coros y orquestas con responsabilidad compartida.

Del análisis realizado en la investigación 2 se desprende el interés por llevar a cabo una coordinación entre los profesores de ambos tipos de centros. Un porcentaje elevado de los directores de las escuelas de música consideran necesaria y beneficiosa una posible coordinación con los maestros de música de la escuela primaria. Sin embargo, a pesar de este interés, el porcentaje de escuelas de música en las que da una coordinación con las escuelas de primaria es bajo.

Por otro lado, existen algunas prácticas programadas por parte de las escuelas de música que favorecen la colaboración con la escuela primaria. Casi la mitad de escuelas de música organiza actividades como audiciones y talleres de instrumentos. Estas actividades se consideran como un primer paso de acercamiento que puede consolidarse en proyectos coordinados de forma conjunta y permanente. De igual modo, el estudio refleja que más de la mitad de las escuelas de música lleva a cabo proyectos conjuntos con otras instituciones comunitarias tanto educativas como sociales.

Estas actividades tienen una repercusión directa en el impacto cultural que ejercen estos centros sobre el municipio. Como afirma Morant (2013), a partir de estas iniciativas se hace extensible el trabajo realizado en los centros al resto de la sociedad. Por ello, conviene incrementar la presencia de estas actividades en la totalidad de las escuelas de música de la Comunidad. Esta colaboración puede representar tanto para el alumnado como para el profesorado una apertura de horizontes, no sólo desde el punto de vista musical, sino también desde el punto de vista de las relaciones humanas. Dada las diferentes situaciones de convivencia y de trabajo que este tipo de colaboraciones generan, la escuela de música necesita dialogar y colaborar con la escuela de primaria y con todas aquellas instituciones o colectivos susceptibles de cooperación (Perrenoud, 2012).

Otra de las conclusiones derivada del estudio se relaciona con el intercambio en foros de trabajo que llevan a cabo los equipos directivos de algunas escuelas de música para contrastar opiniones y experiencias. Estos foros ganarían en importancia si se hacen exten- sibles a los equipos directivos de la escuela primaria con el fin de conocer las buenas prácticas educativas que se llevan a cabo en las escuelas de música y posibilitar así la coordinación de algunas de estas prácticas en la escuela primaria.

Con más de diez años de diferencia, ambas investigaciones convergen en la necesidad de una coordinación real entre la escuela de música, aprendizaje no formal, y la escuela primaria, aprendizaje formal. Los resultados obtenidos contribuyen a fomentar el diálogo que facilite la cooperación entre el profesorado de música de la escuela primaria y de las escuelas de música y aspiran a despertar el interés de todos los sectores implicados a nivel social, cultural y educativo de la comunidad. Por otro lado, acorde con las investigaciones de Riaño $(2008,2010)$ y Morant $(2013)$, las propuestas de coordinación conjunta persiguen el óptimo aprovechamiento de los recursos disponibles con el objetivo de mejorar la calidad de la educación musical.

En este sentido, a tenor de los resultados obtenidos se evidencia que la coordinación entre la escuela de música y la escuela de primaria no es una práctica extendida. A pesar de que el profesorado muestra un interés por querer llevar a cabo proyectos conjuntos y desde las direcciones de los centros se coincide en su necesidad, no se ha atendido a esta realidad educativa de una forma generalizada. Sin embargo, como recogen los trabajos de Botella et al. (2012) y Sierra, Méndez y Mañana (2012), es indudable la necesidad de un trabajo colaborativo y en equipo por parte del profesorado. Se trata de formar al alumnado en la participación en actividades musicales y contribuir así a desarrollar sus capacidades creativas, de expresión y comunicación y en la toma de conciencia del papel de la música en la sociedad actual. La importancia de crear una visión colectiva de la educación debe sustentarse en la configuración de una comunidad educativa capaz de afrontar el reto de aunar esfuerzos y educar juntos (Castro y García, 2013).

Si se desea una mayor coherencia de los objetivos y métodos que van dirigidos a promover el desarrollo individual y social, resulta imprescindible el establecimiento de una sólida alianza entre todas las agencias educativas (Álvarez, 2004). Es en este punto donde se destaca la necesaria coordinación de los centros de educación formal y no formal en torno a la enseñanza de la música. En resumen, se pone de relieve el importante caudal de todo tipo que supone pensar y trabajar conjuntamente distintos profesionales de una misma materia y con un mismo objetivo común, propugnando propuestas didácticas de interacción entre los diferentes medios.

Finalmente, una de las principales limitaciones de este estudio es que ambas investigaciones están centradas en diferentes ámbitos territoriales, auque este hecho no impide aproximarse a la tendencia general del objeto de análisis. Algunas propuestas para futuras investigaciones irían encaminadas a diseñar un instrumento de evaluación común y a su posible aplicación en diferentes realidades territoriales para tener una visión más global y objetiva. Uno de los elementos que conduce a la mejora continuada de las prácticas educativas parte de su evaluación, no con el propósito de demostrar sino de perfeccionar (Stufflebeam y Shinkfield, 1987). El porcentaje de escuelas de música que tienen mecanismos para evaluar la gestión y la incidencia de las colaboraciones externas no llega a una cuarta parte. Por ello, sería conveniente que se incrementara la proporción de centros que se interesa por conocer y evaluar la repercusión de las colaboraciones externas que llevan a cabo como elemento para su mejora.

\section{Referencias bibliográficas}

Álvarez, J. L. (2004). Los escenarios de la educación: espacios formales, no formales, informales. En M. M. Del Pozo (Ed.), Teorías e instituciones contemporáneas de educación (pp. 109-130). Madrid: Biblioteca Nueva.

Berbel, N. (2012). La educación musical elemental en las Illes Balears: evaluación de la calidad de las escuelas de música. (Tesis doctoral). Facultad de Educación de la Universitat de les Illes Balears. 
Botella, A.M., Fernández, R., y Minguez, X. (2012). Interdiciplinarity in arts education: a work proposal. En L. G. Chova, A. L. Martínez e I. C. Torres (Eds.), 5th International Conference of Education, Research and Innovation (ICERI 2012) (pp. 3959-3963). Madrid: International Association of Technology, Education and Development.

Cabedo A., y Díaz, M. (2013). Positive musical experiences in education: music as a social praxis. Music Education Research, 15(4), 455-470. doi:10.1080/14613808.2013 .763780

Campbell, P. S. (1998). Songs in Their Heads. Music and It's Meaning in Children's Lives. New York: Oxford University Press.

Castro, A. M., y García, R. (2013). La visión del profesorado de Educación Infantil y Primaria de Cantabria sobre la participación y las relaciones interpersonales entre los miembros de la comunidad escolar. Aula Abierta, 41(1), 73-84.

Coleman, J. S., y Husen, T. (1989). Inserción de los jóvenes en una sociedad de cambio. Madrid: Narcea.

Colwell, R., y Richardson, C. (2002). The New Handbook of Research on Music Teaching and Learning. New York: Oxford University Press.

Coombs, P. H. (1968). The World Educational Crisis. Nueva York: Oxford University Press, trad. castellana de Solanas, M. (1971), La crisis mundial de la educación. Barcelona: Península.

Coombs, P. H., y Ahmed, M. (1974). Attacking Rural Poverty: how Non-Formal Education can Help. Baltimore: J. Hopkins University Press, trad. castellana de Rodríguez, L. (1975). La lucha contra la pobreza rural. Aporte de la educación no formal. Madrid: Tecnos.

Cremades, R., y Herrera, L. (2010). Estudio comparativo de la educación formal e informal en el conocimiento musical de los estudiantes de enseñanza profesional de música. Publicaciones. Facultad de Educación y Humanidades. Campus de Melilla, 40, 73-87.

Deming, W. E. (1989). Calidad, Productividad y Competitividad. La salida de la crisis. Madrid: Ed. Díaz de Santos.

Díaz, M. (1998). Investigación-acción como soporte para la intervención educativa sistemática: una experiencia. Eufonía, 10, 79-86.

Díaz, M. (2001). La música en la educación primaria y en las escuelas de música: la necesaria coordinación. (Tesis doctoral). Facultad de Filosofía y Ciencias de la Educación de la Universidad del País Vasco.

European Foundation for Quality Management. (1999). Modelo EFQM de Excelencia 1999. Bruselas: European Foundation for Quality Management.

Ibarretxe, G., y Díaz, M. (2008). Music learning into diversified educational systemsur. Revista de Psicodidáctica, 13, 97-110.

Hentschke, L. (2003). A pesquisa em educaçaô musical: Uma aproximaçao entre o ensino formal e a aprendizagem informal de música. En A. Rodríguez (Ed.), Actas de las II Jornadas de Investigación en Educación Musical (pp. 59-72). Granada: Universidad de Granada.

Ministerio de Educación y Cultura. (2001). Modelo Europeo de Excelencia. Adaptación a los Centros Educativos del Modelo de la Fundación Europea para la Gestión de Calidad. Madrid: Secretaría General Técnica.
Mok, O. N. A. (2011). Non-formal learning: clarification of the concept and its application in music learning. Australian Journal of Music Education, 1, 11-15.

Morant, R. (2013). Planteamientos de educación formal en enseñanzas no regladas de música: las Escuelas de Música de las Sociedades Musicales Valencianas. Revista Electrónica de LEEME, 31, 79-106.

North, A. C., y Hargreaves, D. J. (2008). The social and applied psychology of music. Oxford: Oxford University Press.

Oliveira, A. (2003). Articulaçoes de processos pedagógicos e musicais em contextos escolares e nao escolares: estudo de caso na Bahia, Brasil. Música, Psicología e Educaçao do CIPEM, 4, 39-46.

Perrenoud, P. (2012). Cuando la Escuela pretende preparar para la vida. ¿Desarrollar competencias o enseñar otros saberes? Barcelona: Graó.

Riaño, M. E. (2008). La gestión de las actividades musicales del G-9 en contextos de educación formal y no formal. (Serie Tesis doctoral). Bilbao: Servicio Editorial de la Universidad del País Vasco.

Riaño, M. E. (2010). Educación musical formal y no formal en el G9. Eufonía: Didáctica de la Música, 48, 87-98.

Rogers, A. (2004). Looking again at non-formal and informal education - towards a new paradigm. The encyclopaedia of informal education. Recuperado el 12 de julio de 2013 de http: www.infed.org/biblio/non_formal_paradigm.htm

Sierra, B., Méndez, A., y Mañana, J. (2012). Necesidad y propuesta de un procedimiento para programar por Competencias Básicas. Aula Abierta, 40(3), 33-46.

Stufflebeam, D. L., y Shinkfield, A. J. (1987). Evaluación sistemática. Guía teórica y práctica. Barcelona: Paidós/MEC.

Torres, C., y Pareja, J. A. (2007). La educación no formal y diferenciada. Madrid: CCS.

Touriñán, J. M., Rodríguez, A., y Olveira, M. E. (2005). El Espacio Europeo de la Educación Superior: Extensión Universitaria, Convergencia y Orientaciones Axiológicas de carácter global. XXIV Seminario Interuniversitario de Teoría de la Educación "El Espacio Europeo de Educación Superior" (pp. 1-10). Recuperado el 1 de diciembre de 2013 de http: http://pendientedemigracion.ucm.es/info/site/site24.html

Trilla, J. (1993). Otras educaciones: animación sociocultural, formación de adultos y ciudad educativa. Barcelona: Anthropos.

Trilla, J. (1998). La educación fuera de la escuela. Ámbitos no formales y educación social (3.. ed.). Barcelona: Ariel.

Veblen, K., y Olsson, B. (2002). Community Music. En R. Colwell y C. Richardson (Eds.), The handbook of research on music teaching and learning (pp. 730-753). Oxford: New York.

Waldron, J. (2009). Teacher and Student Perceptions of Music Teaching and Learning at the Goderich Celtic College, Goderich, Ontario, Canada. Bulletin of the Council for Research in Music, 182, 51-64.

White, A. M., y Geisler, H. G. (2007). The role of formal music education on children's growth in informal music production. Bulletin of the Council for Research in Music Education, 171, 81-89. 